\title{
Mixed sexually transmitted infections in adult male patients with urethral discharge and female patients with pelvic inflammatory disease
}

\author{
Shamala Moodley \\ Department of Biomedical Sciences, Mangosuthu University of Technology, KwaZulu-Natal, South Africa. \\ Accepted 29 March, 2013
}

\begin{abstract}
To determine the percentage of mixed sexually transmitted infections in a high risk sample population of men and women, urethral and endocervical swabs were collected from 200 men with urethritis and 200 women with Pelvic Inflammatory Disease (PID). Cell cultures, plate culture and microscopy were used to detect the presence of Neisseria gonorrhoeae, Chlamydia trachomatis and Herpes simplex virus (HSV). Serological tests were used to screen for syphilis (Rapid Plasma Reagin-Becton Dickinson) and human immunodeficiency virus (HIV) (Determine, Abbott, USA). The results revealed that $95 \%$ of male participants had a urethral infection and $81 \%$ of female participants had an endocervical infection. In men, $N$. gonorrhoea (84\%) was the major causative organism followed by Chlamydia trachomatis (65.5\%). The most frequently occurring causative organism in women was $C$. trachomatis $(72 \%)$ followed by $N$. gonorrhoeae $(22 \%)$. The sample population revealed high rates of sexually transmitted infections. There were significant associations between STIs and continued high risk sexual practices in men and women. These findings support the need for studies that confirm the percentage of sexually transmitted infections (STIs) using clinical laboratory tests.
\end{abstract}

Key words: Sexually transmitted infections, human immunodeficiency virus, sexual risk, endocervical, urethral.

\section{INTRODUCTION}

Sexually Transmitted Infections (STIs) present a major public health concern in both industrialised and developing countries. However, information about infection rates is limited, especially for many developing countries (Thior et al., 1997; Gertig et al., 1997).

Together with human immunodeficiency virus (HIV), STIs result in substantial morbidity and mortality (Thior et al., 1997; Gertig et al., 1997; Wasserheit, 1992; Mayaud et al., 1995); Neisseria gonorrheae, the organism most frequently concomitant with Chlamydia trachomatis infections, that involve the lower genital tract through direct infection of the columnar epithelium of mucosal membranes (Afrakhteh et al., 2008). C. trachomatis infections are the most recognized causes of salpingitis with a sequelae of chronic pelvic inflammatory disease leading to infertility and ectopic pregnancies in women and sterility in men (Hoosen et al., 1989, 1993).

The present study was undertaken to determine the percentage of mixed infections in men with urethral discharge and women with pelvic inflammatory disease (PID). 


\section{MATERIALS AND METHODS}

\section{Patients}

This is a retrospective study carried out over a period of two years (2006 to 2007). During this period, patients were recruited, specimens collected and all analyses performed. A total of 200 adult black male patients attending the city health, sexually transmitted infection (STI) clinic in Durban, South Africa, were recruited. Men with complaints of urethral discharge were included in the study. The female patient population consisted of 200 black women attending the Gynaecology Out-patient Department (GOPD) at a regional hospital situated in Kwa-Zulu Natal, South Africa. All female patients presented with abdominal complaints and in whom a clinical diagnosis of PID was made by the attending clinic doctor were recruited in the study. Both male and female patients who had received antibiotics within the previous two weeks were excluded from the study.

Informed consent was obtained from all participants. The study was approved by the University of KwaZulu Natal Ethics Committee.

\section{Specimen collection}

\section{Swabs}

Four endourethral swabs were collected from all male patients. The swabs were consecutively inserted 2 to $4 \mathrm{~cm}$ into the urethra and the urethral mucosa was gently scraped. Smears were made, with the first swab, on clean slides for Gram stain and microscopy. Modified New York City (NYC) medium was thereafter, inoculated with the second swab for the isolation of $N$. gonorrhoeae. The NYC plates were streaked and immediately placed into a candle jar, with $\mathrm{CO}_{2}$. Swabs collected thereafter, were placed separately into Chlamydia transport medium (CTM) for $C$. trachomatis and another into viral transport medium (VTM) for herpes simplex virus (HSV) culture. Similar number of endocervical specimens were collected and processed as follows: Sterile cotton- tipped swabs (plastic shaft) were sequentially inserted into the endocervical canal and rotated firmly to obtain secretions and cervical cells. The swabs were processed and transported in the same manner as the male endouretheral specimens.

\section{Serological specimens}

Whole blood (5 to $10 \mathrm{ml}$ ) was collected from each patient. The serum was separated aseptically and tested for antibodies to HIV (Determine, Abbott, USA) and for syphilis, using a treponemal antibody assay.

\section{Transport and storage of specimens}

All CTM and VTM samples were held at $4^{\circ} \mathrm{C}$ on ice, and transported on ice, to the laboratory within 2 to $4 \mathrm{~h}$ of collection. NYC agar plates, which were inoculated at the site of specimen collection, were placed into candle extinction jars and thereafter transported to the laboratory for incubation at $37^{\circ} \mathrm{C}$ in $6 \% \mathrm{CO}_{2}$ atmosphere. On arrival at the laboratory, specimens in CTM were inoculated onto prepared McCoy cell monolayers for the isolation of $C$. trachomatis and the VTM specimens were cultured onto monolayers of Green monkey kidney cell lines for HSV.

\section{Laboratory methods}

\section{Cell culture for C. trachomatis}

All specimens were cultured, in duplicate, onto monolayers of cyclo- heximide treated McCoy cells in shell vials containing $12 \mathrm{~mm}$ round coverslips (Sterilin) and growth medium (Eagles Minimum Essential Medium with $10 \%$ foetal calf serum). The shell vials were incubated for $72 \mathrm{~h}$ at $37^{\circ} \mathrm{C}$ in $5 \% \mathrm{CO}_{2}$ atmosphere. After incubation, the medium in each vial was aspirated and the coverslips were fixed in $10 \%$ methanol, air dried, mounted onto clean slides and stained with immunofluorescent stain according to manufacturer's instructions (Syva Co,USA). The slides were examined for $C$. trachomatis at $40 \mathrm{x}$ magnification and morphology confirmed at 100x (oil immersion) magnification (Olympus Fluorescence Microscope, $\mathrm{BH} 2)$. Positive and negative control slides were stained with each batch of slides so as to verify the quality of the stain.

\section{Culture for Herpes simplex virus}

The presence of Herpes simplex virus in urethral and endocervical specimens was detected by culture in vervet monkey kidney cells. Briefly, $200 \mu \mathrm{l}$ of supernatant was inoculated onto cell monolayers, in duplicate. The inoculated cells were incubated at $37^{\circ} \mathrm{C}$ and observed daily for cytopathetic effects (CPE), for up to 14 days. The observation of characteristic CPE was considered a positive result and the presence of HSV was confirmed by staining with fluorescein isothiocyanate labelled monoclonal antibody (MA Bioproducts) against HSV (Types 1 and 2).

\section{Direct smear examination: Gram stain}

A direct Gram stain was performed on all smears made at the site of specimen collection. Air dried slides were heat fixed and stained using the standard Gram stain technique. Thereafter, slides were examined using 100x (oil immersion) objective, for the presence of pus cells, typical intracellular Gram negative diplococci and other bacteria.

\section{Culture for $N$. gonorrhoeae}

Modified New York City agar (Becton- Dickenson, USA) were directly incubated at the site of specimen collection and incubated for $48 \mathrm{~h}$ at $37^{\circ} \mathrm{C}$ in $6 \% \mathrm{CO}_{2} . \mathrm{N}$. gonorrhoeae colonies were identified by their colonial morphology, as Gram negative diplococci which were oxidase positive (Organon Technica). The kidney shaped organism was confirmed by the carbohydrate fermentation test (Becton- Dickenson, USA). Isolates were screened for penicillinase production, using chromogenic cephalosporin (nitrocephin- $0.5 \% \mathrm{w} / \mathrm{v}$ ) as substrate (Oxoid).

\section{Serological methods}

\section{Syphilis}

The non-specific, rapid plasma reagin (RPR) test (Becton Dickson) was used as a screening test for the detection of non-specific antibodies to syphilis. The test was performed according to manufacturer's instructions.

Semi quantitative tests using RPR reagent, using serially diluted serum, were performed on serum specimens, which were positive on the qualitative screening test. The Treponema pallidum haemagglutination antibody test (TPHA), specific for syphilis, was performed on all sera according to manufacturer's instructions (Omega Diagnostics). This was done to confirm all positive RPR results and to give an indication of the number of patients with past exposure to syphilis infections. Agglutination of test cells but not control cells was indicative of the presence of specific antibody to T. pallidum. 
Table 1. Overall positivity of sexually transmitted pathogens in men with urethritis and women with pelvic inflammatory disease.

\begin{tabular}{lcc}
\hline \multirow{2}{*}{ Pathogen } & Men = no. 200 & Women = no. 200 \\
\cline { 2 - 3 } & Positive (\%) & Positive (\%) \\
\hline N. gonorrhoeae (Culture) & $168(84.0)$ & $44(22.0)$ \\
Gram stain & $146^{*}(73.0)$ & $34^{*}(17.0)$ \\
ß-Latamase positive strains & $17^{\circ}(10.1)$ & $7^{*}(22.0)$ \\
C. trachomatis & $131(65.5)$ & $144(72.0)$ \\
Serology & & \\
Syphilis & $27(13.5)$ & \\
HIV & $82(41.0)$ & $43(21.5)$ \\
HSV (Type II) & $0(0)$ & $75(37.5)$ \\
\hline
\end{tabular}

* All 146 and 34 Gram stained smears which showed Gram negative, intracellular, diplococci on microscopy were grown on culture (from men with urethritis and women with PID, respectively). 'Represents $17 / 168$ and $7 / 44$ culture positive $N$. gonorrhoeae results, from men with urethritis and women with PID respectively.

The indirect fluorescent treponemal antibody absorption (FTA-Abs) test using treponemal antigen, sorbent and fluorescein labeled antihuman immuglobulin conjugate (BioMereux) was used as a specific test for confirmation of syphilis in which TPHA reading pattern could not be conclusively interpreted.

\section{Human immunodeficiency virus}

Patients sera were tested for antibodies to HIV-1 (Determine, Abbott, USA) and Vironostika HIV (Uni-form II Organon Teknika). If sera tested positive on both assays, the patients were considered positive. Discrepant results between the first and second tests were further tested by Western blot (Biotechnology Diagnostics).

\section{Statistical analysis}

SPSS statistical software was used for all descriptive analyses in the study. Correlations were assessed using Pearson's correlation test. A $p$-value $<0.05$ was considered statistically significant.

\section{RESULTS}

\section{Demographic data of patients}

The mean age of all men in the study was 34.5 years (range: 15 to 55 years). Of the 200 men, 165 (82.5\%) were single and $35(17.5 \%)$ were married. Multiple sexual partners were recorded in $180(90.0 \%)$ male patients, whilst $20(10.0 \%)$ stated that they were in a single partner relationship. The mean number of lifetime sexual partners was 4.

The mean age of the women was 36.5 years with a range of 15 to 60 years. Single status was reported by $176(88.0 \%)$ female patients whilst $24(12.0 \%)$ were married. Of the 200 women, $95(47.7 \%)$ claimed to have single lifetime partners. The remaining 105 (52.5\%) had multiple partners. The mean number of lifetime partners was 3 . There were 11 more participants who were single amongst the female patients. However, analyses revealed that $37.5 \%$ more males claimed multiple relationships.

\section{Men with urethritis}

Data representative of all sexually transmitted pathogens screened for in this study is shown in Table 1. The criteria for diagnosis of chlamydial infection was based on immunofluorescent stained culture slides. For $N$. gonorrhoeae and Herpes simplex virus, the culture results were considered positive. Serological tests for syphilis were considered positive if both RPR and TPHA were positive. HIV diagnosis was based on positive results of two assays confirmed by FTA-Abs technique or Western Blot.

Urethral smears from males with symptomatic gonorrhoea contained intracellular Gram negative diplococcic in polymorphonuclear leucocytes (PMLs). Multiple infections of sexually transmitted pathogens were diagnosed in $137(72 \%)$ of the male sample population. Of the 200 patients, $190(95.0 \%)$ had a urethral infection. A combination of $3(1.6 \%)$ patients had urethral infections as well as antibodies to syphilis and HIV, whilst $N$. gonorrhoeae and $C$. trachomatis was seen in $111(58.5 \%)$ patients (Figure $1 \mathrm{~A})$. There was a significant association with $N$. gonorrhoeae and number of sexual partners encountered $[p=0.03$; odds ratio (OR), 3.85]. Antibodies to HIV only, were detected in 6 (3\%) patients. The most common infecting organism in this study population was N. gonorrhoeae (84.0\%) followed by $C$. trachomatis $(65.5 \%)$. Syphilis was detected in $13.5 \%$, whilst $41.0 \%$ were HIV positive. Altogether, 146 of 168 (86.9\%) culture positive urethral exudates revealed Gram negative diplococcic morphologically resembling $N$. gonorrhoeae on Gram stained smears. This gave a sensitivity of $87 \%$. All the $146 \mathrm{Gram}$ 


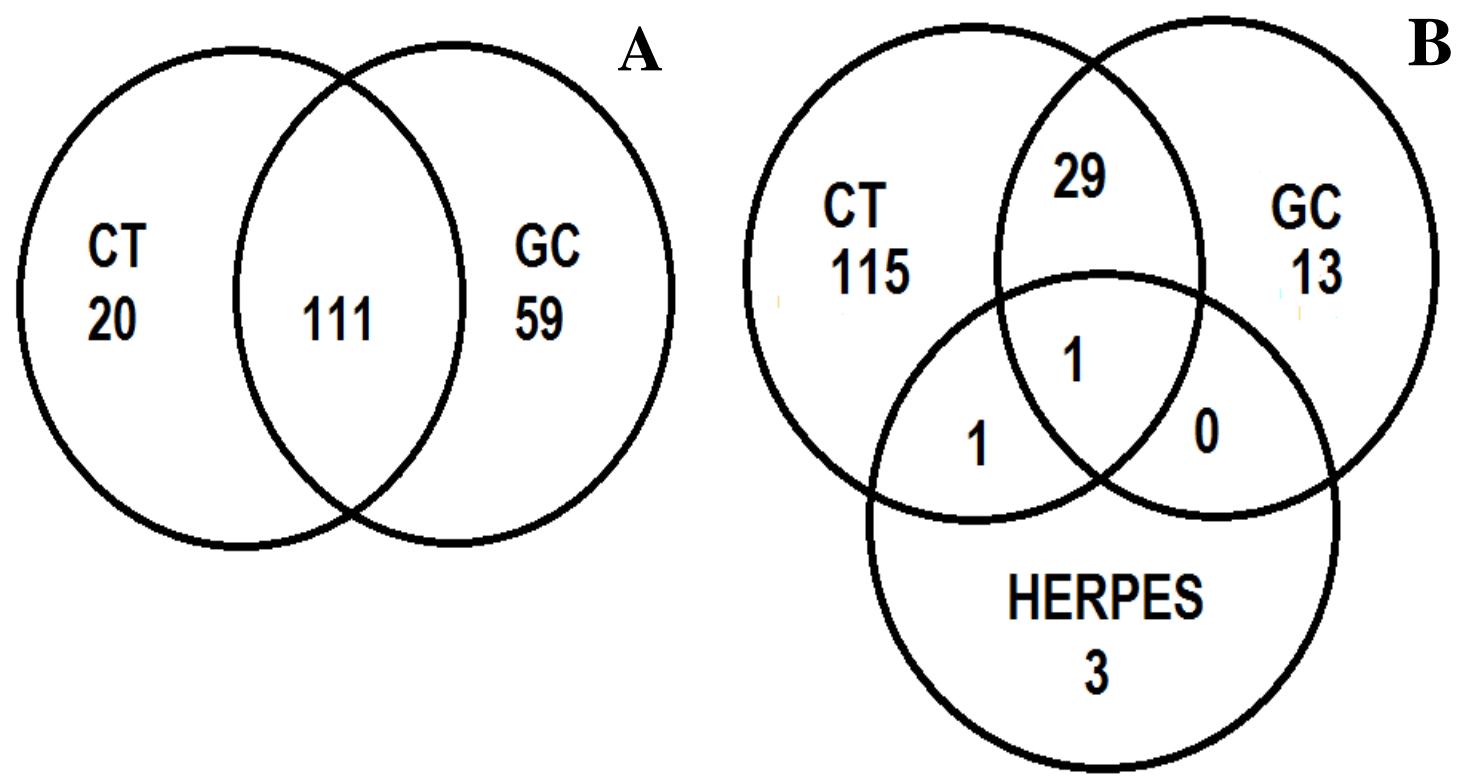

Figure 1. Single and mixed infections in men with urethritis $(A)$ and women with pelvic inflammatory disease (B). $\mathrm{CT}=$ C. trachomatis; $\mathrm{GC}=N$. gonorrhoeae.

Table 2. Human immunodeficiency virus status in men and women with mixed infections.

\begin{tabular}{lcccc}
\hline \multirow{2}{*}{ Pathogen } & \multicolumn{2}{c}{ Men (no. = 200) } & \multicolumn{2}{c}{ Women (no. = 200) } \\
\cline { 2 - 5 } & HIV (+) & HIV(-) & HIV (+) & HIV (-) \\
\hline N. gonorrhoeae & 21 & 38 & 4 & 9 \\
C. trachomatis & 6 & 14 & 61 & 54 \\
N. gonorrhoeae and C. trachomatis & 42 & 69 & 13 & 17 \\
Syphilis antibodies & 14 & 13 & 18 & 25 \\
\hline
\end{tabular}

stained $N$. gonorrhoeae positive smears grew on the culture. Beta-lactamase producing strains were observed in $17(10.1 \%)$ culture positive $N$. gonorrhoeae isolates.

\section{Women with pelvic inflammatory disease}

The reference method for diagnosis of $C$. Trachomatis, $N$. gonorrhoeae, HSV, syphilis and HIV were the same as that used for the male in this study. More than one type of sexually transmitted pathogen was detected in $50 \%$ of the female population studied (Figure 1B). A total of 162 $(81.0 \%)$ women had an endocervical infection. Thirty one $(19.1 \%)$ of whom, had mixed infections. Concurrent gonococcal and chlamydial infection was seen in 29 (17.9\%) patients. Three (1.9\%) had mixed infections which included $C$. trachomatis, $N$. gonorrhoeae, syphilis and HIV-1. There were only 15 (9.3\%) and 7 (4.3\%) patients who had no other infection except serological tests positive for syphilis and antibodies to HIV, respectively. Chlamydial infection was the commonest with $72 \%$ of women infected. As determined using logistic regression analysis, risk factors (PID) for multiple partners, was significant $[p=0.04$; odds ratio $(\mathrm{OR}), 3.8$; 95\% confidence interval (Cl), 1.06 to 13.9]. Gonococcal infection was found in $22 \%$ of the study population. All gonococcal isolates were tested for beta-lactamase production. Beta-lactamase was found in 7 of the 44 (16.0\%) isolates. Characteristic Gram negative, intracellular diplococcic were seen in only $34(17.0 \%)$ Gram stained cervical exudate smears (Table 1). This gave a sensitivity of $77.3 \%$ (34 of 44 ).

\section{Mixed infection and human immunodeficiency virus}

Ninety three percent of men (69 of 74) and $92 \%$ women (78 of 85 ) who had antibodies to HIV had concomitant mixed infections (Table 2). C. trachomatis and $N$. gonorrhoeae were detected in $70 \%$ (80 of 115) of women and $96 \%$ (121 of 126) of men did not demonstrate antibodies to HIV. There were no significant differences 
between patients who had gonococcal and/or chlamydial infections and the presence/or absence of antibodies to HIV-1. However, the men demonstrated a significantly higher percentage of sexually transmitted infections than women.

\section{DISCUSSION}

The findings in this study highlight the need for continuous monitoring and syndromic management of STIs. More specifically, this study's data shows that mixed gonococcal/chlamydial infections account for up to $95 \%$ of STI clinic males, and $78.5 \%$ of women with PID. At the site of collection, a presumptive diagnosis of $N$. gonorrheae was made based on observation of Gramnegative intracellular diplococci in Gram-stained smears of urethral discharges from men and endocervical secretions from women. Confirmatory identification, using biochemical techniques, revealed that $85 \%$ men with urethritis and $21.5 \%$ women with PID were infected with N. gonorrhoeae. Internationally, the range is between 11 and $38.2 \%$ (Creighton et al., 2003). The findings in this study differ from other studies (Garland et al., 2001; Duda et al., 2005; Abdel-Hamid et al., 2008).

Prevalence of chlamydial infection rates varies amongst women, globally. Most pregnant women tested positive in Europe from $2.7 \%$ in Italy to $8 \%$ in Iceland. In Africa, reports in pregnant women ranged from 6 to $13 \%$ and $2.1 \%$ in Brazil (World Health Organization, 2001; Gerbase et al., 1998). However, these studies were done in pregnant women. The study under consideration observed $73 \%$ non pregnant women, with PID, infected with $C$. trachomatis.

To the best of the current knowledge, literature on data for PID in non pregnant women, remain limited. The isolation of $C$. trachomatis infection in KwaZulu-Natal, statistically, is relatively high. Considering the males, the infection rate was $65.5 \%$ when compared with other subSaharan Africa, of 15 to $20 \%$ (World Health Organization, 2001). Together, with Mongolia (14\%) and the UK $(18.8 \%$ ), a vast difference is noted (Garland et al., 2001; Rastogi et al., 2003). Overall, the values were higher in this study when compared with other studies. One explanation for this phenomenon may be the high risk of acquiring a STI when diagnosed with HIV. C. trachomatis appeared relatively minimal between men with urethritis and women with PID (6.5\%). However, there was a marked difference between men and women who had $N$. gonorrhoeae (62\%).

Sexually transmitted diseases such as $N$. gonorrhoeae, C. trachomatis, Herpes and syphilis can increase the risk of HIV transmission. It is also well documented that HIV negative individuals who have an STI are at increased risk of becoming infected with HIV-1 (Wilson et al., 2010; Kissinger et al., 2012). This study reports that $41 \%$ males and $37.7 \%$ females were HIV positive and were co-infected with other STIs. These infections are likely to be particularly important in promoting the sexual transmission of HIV-1 and should therefore, be the focus of HIV prevention strategies.

Studies conducted by De Muylder et al. (1990) revealed that women with PID, infertility and tubal disease had significantly higher prevalence of $C$. Trachomatis antibodies. This is in accordance with the sample population in this study of female GOPD attendees. The risk factors that increase the potential for exposure to infected sex partners, and thus infection, include the number of multiple sex partners over the individual's lifetime (Doherty et al., 2005). Further, contributing factors could be the magnitude of the epidemic, the potentially high risk in number of sexual contacts and the abstinence of condoms, particularly with men.

In total, the culture of Herpes simplex virus demonstrated only 7 women that were infected. It is evident that fewer numbers of the female sample population was HSV-2 positive (2.5\%) when compared with 20 to $40 \%$ of their Central American counterparts (Weiss, 2004). The male patients appeared to be less at risk of acquiring HSV-2 than females.

Based on the findings of the analyses, the prevalence of syphilis was seen in two sets of patients. Patients, both males and females, were either HIV positive with a syphilis infection (42.7 and $46.6 \%$ ) or HIV negative with syphilis (28 and $38.4 \%$ ), respectively. There was a marginal difference between the males when compared with the females with HIV and without HIV.

It is evident that $N$. gonorrhoeae and $C$. trachomatis are the leading causes of STI infection. The risk associated with other STIs is further complicated with HIV. Further studies are necessary to determine changes in the relationship of STDs and HIV infection in the population. Whilst infections in this study are fairly comprehensive, it is also probably true for developing countries, that there are at present no reliable statistics on the true prevalence of sexually transmitted infections. The correct diagnosis and treatment for these diseases are inadequate in developing countries, where major public health services are underfunded and over utilised. The need for control of STI is urgent, since it is recognised as independent risk factors for the acquisition of HIV. Failure to diagnose and treat infections such as $N$. gonorrhoeae, $C$. trachomatis and syphilis can have major health and social challenges, in both male and female infertility (Mostafa and Roshdy, 1999). Various areas of concern are the deleterious effect on pregnancy, neonate and other complications, particularly in women, such as pelvic inflammatory disease and ectopic pregnancy (Ravi and Nair, 2004). Therefore, there is need for formal monitoring and surveillance of STIs.

\section{Limitations of the study}

A notable limitation of this study was STIs within a "high 
risk population" in men and PID in women. High risk populations have a special access-related need, such as a clinic for sexually transmitted infections only. There is need for a survey with various age categories and specific infections such as urethritis, chancroid, genital ulcers, mucopurulent cervicitis, proctitis, for both men and women. The routine coverage estimations would perhaps be more accurately reported. When dealing with patients from an STI clinic, there would be an over reporting of infections, compounded with high HIV estimates. An additional limitation to the study would be the limited screening of specific STIs, when compared with a wider range. However, the task would require the logistics of wider screening and a large human resource component.

\section{ACKNOWLEDGEMENTS}

This study was supported by the National Research Foundation (SA). Dr M. E. Essa and her staff are appreciated for their assistance in specimen collection. The cooperation and consent of the patients in this study is appreciated. Grants support for the study was received from Mangosuthu University of Technology Research Funding.

\section{REFERENCES}

Abdel-Hamid F, El-Gamal, Sultan RS, Al Otaibi, Alshamali A, Abdulrazzaq A (2008). Mixed urethral Infection in patients with urethral discharge in the southern region of Kuwait. Indian J. Sex. Transm. Dis. 29(1):29-31.

Afrakhteh M, Beyhaghi H, Moradi A, Hosseini SJ, Mahdavi A, Giti S (2008). Sexually Transmitted Infections in Tehran. J. Fam. Reprod. Health 2(3):123-128.

Creighton S, Tenant-Flowers M, Taylor CB, Miller R, Low N (2003). Coinfection with gonorrhoea and chlamydia: how much is there and what does it mean? Int. J. STD AIDS 14(2):109-113.

De Muylder X, Laga M, Tennstedt C, Van Dyck E, Aelbers GN, Piot P (1990). The role of Neisseria gonorrhoeae and Chlamydia trachomatis in pelvic inflammatory disease and its sequelae in Zimbabwe. J. Infect. Dis. 162(2):501-505.

Doherty IA, Padian NS, Marlow C, Aral SO (2005). Determinants and Consequences of Sexual Networks as They Affect the Spread of Sexually Transmitted Infections. J. Infect. Dis. 191(1):42-54.

Duda RB, Darko R, Adanu,RMK , Seffah J, Anarfi JK, Gautam S et al (2005). HIV prevalence and risk factors in women of Accra, Ghana: results from the women's health study of Accra. AM. J. Trop. Med. Hyg. 73(1):63-66.
Garland SM, Tabrizi SN, Chen S, Byambaa C, Davaajav K (2001). Prevalence of Sexually Transmitted Infections (Neisseria gonorrhoeae, Chlamydia trachomatis, Trichomonas vaginalis and Human Papillomavirus) in Female Attendees of a Sexually Transmitted Diseases Clinic in Ulaanbaatar, Mongolia. Infect. Dis. Obstet. Gynecol. 9(3):143-146.

Gerbase AC, Rowley JT, Heymann DH, Berkley SF, Piot P (1998). Global prevalence and incidence estimates of selected curable STDs. Sex. Transm. Infect. 74(1):12-16.

Gertig DM, Kapiga SH, Shao JF, Hunter DJ (1997). Risk factors for sexually transmitted diseases among women attending family planning clinics in Dar-es-Salaam, Tanzania. Genitourin Med. 73(1):39-43.

Hoosen AA, N O'Farrell, Van den Ende J (1993). Microbiology of acute epididymitis in a developing community. Genitourin Med. 69(5):361-363

Hoosen AA, Quinlan DJ, Moodley J, Kharsany ABM, Van den Ende J (1989). Sexually transmitted pathogens in acute pelvic inflammatory disease. S. Afr. Med. J. 76:251-254.

Kissinger P, Kovacs S, Anderson-Smits C, Schmidt N, Salinas O, Hembling $\mathrm{J}$ (2012). Patterns and predictors of HIV/STI risk among Latino migrant men in a new receiving community. AIDS Behav. 16(1):199-213.

Mayaud P, Grosskurth H, Changalucha J, Todd J, West B, Gabone R (1995). Risk assessment and other screening options for gonorrhoea and chlamydial infections in women attending rural Tanzanian antenatal clinics. Bull. World Health Organ. 73(5):621-630.

Rastogi S, Das B, Salhan S, Mittal A (2003). Effect of treatment for Chlamydia trachomatis during pregnancy. Gynae. Obstet. 80(2):129137.

Mostafa SR, Roshdy OH (1999). Risk profiles for sexually transmitted diseases among patients attending the venereal disease clinic at Alexandria Main University Hospital. J. Eastern Medit. Health $5(4): 740-754$

Ravi R, Nair SB (2004). Correlates of sexually transmitted infections among women in southern India. J. Fam. Welfare 57(1):45-54.

Thior I, Diouf G, Diaw IK, Sarr AD, Hsieh CC, Ndoye I, Mboup S, Chen L, Essex M, Marlink R, Kanki P (1997). Sexually transmitted diseases and risk of HIV infection in men attending a sexually transmitted diseases clinic in Dakar, Senegal. Afr. J. Reprod. Health 1(2):26-35.

Wasserheit JN (1992). Epidemiological synergy. Interrelationship between human immunodeficiency virus infection and other sexually transmitted diseases. Sex. Transm. Infect. 19(2):61-77.

Weiss $\mathrm{H}$ (2004). Epidemiology of herpes simplex virus type 2 infection in the developing world. Herpes 11(1):24-35.

Wilson KS, Eggleston E, Diaz-Olavarrieta C and Garcia SG (2010). $\mathrm{HIV/STI}$ risk among male Mexican immigrants in Dallas, Texas findings from a pilot study. J. Immigr. Minor. Health 12(6):947-951.

World Health Organization (2001). Global Prevalence and Incidence of Selected Curable Sexually Transmitted Infections: Overview and Estimates, Geneva: WHO, WHO/HIV_AIDS. 